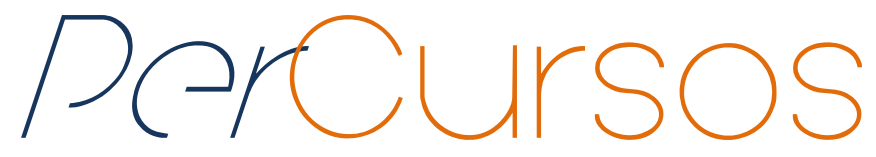

\title{
Mobilização social para adaptação à mudança climática: uma experiência na bacia do Rio Pardo (RS)
}

\section{Resumo}

Face à inatividade de governos nacionais em promover adaptação à mudança do clima, a adaptação pela esfera subnacional vem ganhando relevância no debate acadêmico. Na diversidade de iniciativas inovadoras de adaptação subnacional no âmbito da federação brasileira, a legislação adotada no Rio Grande do Sul recebeu destaque. As ferramentas para a Política Gaúcha de Adaptação Climática, porém, (ainda) não estão operacionais. O presente trabalho sintetiza resultados de projeto de pesquisa aplicada, entre 2016 e 2017, que visa identificar e dar visibilidade a inovações na adaptação adotadas de forma voluntária por atores privados e não governamentais na Região Central do RS. Foram identificadas cerca de uma centena de organizações, entre empresas de pequeno e grande porte, cooperativas, universidades e órgãos públicos locais, que estão testando mecanismos para compensar suas emissões de gases de efeito estufa, promovendo a eficiência energética ou o pagamento por serviços ambientais. O projeto aqui descrito visa a contribuir para registro, análise e difusão dessas inovações, entendidas como iniciativa de divulgação científica pela universidade comunitária UNISC para o debate e a mobilização social na esfera regional.

Palavras-chave: Mudança Climática. Adaptação Climática. Vale do Rio Pardo (Brasil).

\author{
Markus Erwin Brose \\ Doutor em Sociologia Política \\ pela Universidade de \\ Osnabrück/Alemanha. \\ Professor da Universidade de \\ Santa Cruz do Sul - UNISC/RS. \\ Brasil \\ markus@unisc.br
}

\section{Para citar este artigo: \\ BROSE, Markus Erwin. Mobilização social para adaptação à mudança climática: uma experiência na bacia do Rio Pardo (RS). Revista PerCursos, Florianópolis, v. 19, n.39, p. 291 - 306, jan./abr. 2018.}

DOI: 10.5965/1984724619392018291

http://dx.doi.org/10.5965/1984724619392018291 


\title{
Social mobilization for climate change adaptation: an experience in the Pardo River basin/Brazil
}

\begin{abstract}
Given the inaction of many national governments to promote adaptation to climate change, adaptation through the subnational sphere has gained relevance in academic debate. Within the diversity of innovative initiatives of subnational adaptation in the Brazilian federation, the legislation adopted by Rio Grande do Sul state received special attention. The state Climate Adaptation Policy, however, (still) is not operational. The present work synthesizes results of an applied research project, which aims to identify and give visibility to innovations in adaptation adopted voluntarily by private and non-governmental actors in the Central Region of RS. Some 100 organizations have been identified, ranging from small to large companies, cooperatives, universities and local public agencies that are offsetting their greenhouse gas emissions, promoting energy efficiency or testing payment for environmental services. The project aims to contribute to the registration, analysis and dissemination of these innovations, understood as an initiative by the community college UNISC to foster the debate and social mobilization at the regional level.
\end{abstract}

Keywords: Climate Change. Climate Adaptation. Rio Pardo Valley (Brazil). 


\section{Introdução}

O Painel Intergovernamental sobre Mudanças Climáticas (IPCC, em inglês) sintetiza o consenso da comunidade científica sobre origens e impactos da mudança do clima mediante ciclos de revisão da produção bibliográfica especializada. Contribui, assim, ativamente para enriquecer o debate público e, indiretamente, para a formulação de políticas públicas. O IPCC, financiado pelos Estados membros das Nações Unidas (GAO, 2011), privilegia uma perspectiva estatal, priorizando, portanto, governos nacionais como destinatários de suas recomendações. O contínuo crescimento global das emissões de gases de efeito estufa (BODEN; MARLAND; ANDRES, 2017), porém, contribui para gerar dúvidas sobre qual tem sido o efeito concreto das recomendações do IPCC aos governos nacionais.

O quinto relatório do IPCC, publicado em 2014, dedica um capítulo para analisar o papel dos governos subnacionais na adaptação à mudança do clima (SOMANATHAN; STERNER; SUGIYAMA, 2014), capítulo este originado da crescente relevância conferida à inovação pelos governos subnacionais na pesquisa especializada (CUNHA; REI; WALTER, 2009; GALARRAGA; GONZALEZ; MARKANDYA, 2011; GALARRAGA; SAINZ; FRANÇA, 2017). Adaptação foi entendida como: "ajustes em sistemas naturais ou sociais em resposta à mudança climática e seus impactos esperados, prevenindo riscos ou aproveitando novas oportunidades"1 (BAUER; STEURER, 2014, p. 1).

A capacidade de inovação pelos atores subnacionais vem sendo evidenciada, por exemplo, pela Califórnia, devido à adoção, em 2006, de legislação e das políticas públicas consideradas o estado da arte em adaptação, em oposição às posições ambíguas, ou mesmo conflituosas, do governo federal dos EUA quanto à mudança climática (BROSE, 2013). Esse pioneirismo da Califórnia contribuiu para que outros atores subnacionais incluindo estados brasileiros - decidissem formar a Força Tarefa dos Governadores para Clima e Florestas (GCF Task Force, em inglês). Neste contexto, dada sua dimensão geográfica e grau de descentralização, o Brasil pode ser interpretado como um laboratório vivo para a comparação e análise de iniciativas subnacionais de adaptação:

\footnotetext{
${ }^{1}$ Esta, e demais citações, originalmente em Inglês, constituem tradução livre do autor.
} 
desde o pioneirismo de municípios que realizaram inventários das emissões de gases de efeito estufa de suas economias (NOBRE et al., 2010), até aqueles que realizam oposição a essas políticas, em forma de subsídios oferecidos por governos municipais e estaduais para atrair termelétricas movidas a óleo diesel ou carvão mineral.

Mapeamentos das iniciativas subnacionais de adaptação no Brasil vêm proporcionando destaque ao estado do Rio Grande do Sul (RS). Tanto em estudos acadêmicos (ANTUNES; SANCHEZ, 2013; BARBI, FERREIRA, 2016), como em análise pelo Governo Federal (ROMEIRO; PARENTE, 2011) ou mapeamento fomentado pelo Banco Mundial (PAGIOLA; GLEHN; TAFFARELLO, 2013), o RS foi citado como exemplo de inovação subnacional na adaptação. A Rede de Governos Regionais para o Desenvolvimento Sustentável (NRG4SD, em inglês), criada durante a Conferência Global do Meio Ambiente, na África do Sul, lista o RS como membro-fundador.

O destaque conferido ao RS - um estado periférico ao eixo RJ/SP para inovações em políticas públicas - parece decorrer da sua abrangente legislação. O Decreto n. 45.098, de 15/06/07 instituiu o Fórum Gaúcho de Mudanças Climáticas, que contribuiu para a formulação da lei n. 13.594, de 30/12/10, estabelecendo a Política Gaúcha sobre Mudanças Climáticas (PGMC). Essa legislação prevê o Fundo Estadual de Mudanças Climáticas, a Secretaria Estadual da PGMC, além do Registro Público de Emissões de Gases de Efeito Estufa, um conjunto de ferramentas que almeja promover a descarbonização da economia regional. Decorrida uma década, porém, estas iniciativas não estão operacionais. A PGMC alinha-se a um fenômeno comum no país: as legislações promulgadas, mas, não implementadas. Entre a inoperância do Governo Federal, que assiste ao crescimento exponencial do desmatamento na Amazônia, descaracterizando o Plano Nacional de Adaptação (BRASIL, 2015), e o default do Governo do RS, coloca-se a questão: de onde pode surgir a inovação gerencial e tecnológica para adaptação à mudança do clima?

Em manifesto pela maior relevância aos aspectos políticos da mudança climática, o sociólogo Ulrich Beck, proponente do conceito da Sociedade de Risco, enfatizou: 
Sem uma maioria composta por grupos sociais muito diversos, que não apenas falem sobre, mas que atuem e votem a favor de políticas públicas relacionadas ao clima [...] a ação pública não terá futuro. Apenas se encontrarmos resposta à questão urgente e pouco debatida: de onde virá o apoio cotidiano da população, o suporte pelas mais diversas classes, diferentes nações e ideologias, que são afetadas de forma diversa pela mudança climática? Somente então as políticas de mudança climática perderão seu status elitista. (BECK, 2010, p. 255)

Beck enfatiza, assim, a necessidade de maior informação e engajamento no debate público sobre a mudança climática, considerado um dos riscos centrais da atualidade. Argumento este que vem ao encontro dos resultados de pesquisa de opinião encomendada, apenas quatro anos mais tarde, pelo Ministério da Ciência e Tecnologia quanto ao interesse da população brasileira em divulgação científica (CENTRO DE GESTÃO E ESTUDOS ESTRATÉGICOS, 2017). No quesito “Percepções de risco e questões socioambientais no Brasil”, o maior grau de preocupação dos entrevistados foi com “desmatamento na Amazônia”, em segundo lugar "efeitos da mudança climática”. Os autores do relatório sugerem que estas respostas não estão associadas ao medo pela população, mas "a uma preocupação que alimenta um comprometimento e um interesse por uma maior apropriação e participação nessas temáticas e discussões" (CGEE, 2017, p. 85). Parafraseando Beck: De onde surgem inovações na adaptação à mudança climática que possam subsidiar o debate público?

Mediante revisão do debate internacional, grupo de pesquisadores do Centro Tydall de Pesquisa em Mudança Climática no Reino Unido (JORDAN; HUITEMA, 2014) confirmam a existência de um viés estatal em ampla parcela da bibliografia e questionam: “Chama atenção o fato da pouca bibliografia disponível sobre adaptação subnacional” (p. 393). Segundo os autores, o Estado dispõe da legitimidade e dos recursos (jurídicos, financeiros) necessários para desenvolver visões de longo prazo, que possibilitam mudanças estruturais para a descarbonização da economia; mas, por default, em muitas situações são agentes privados e organizações não estatais que estão promovendo inovações (que, posteriormente, podem ser adotadas em maior escala em políticas públicas). 
Diante da diversidade de iniciativas não estatais na bibliografia sobre adaptação subnacional, Jordan e Huitema (2014) propõem uma tipologia para interpretar inovações (Quadro 1).

Quadro 1 - perspectivas sobre inovação na adaptação climática

\begin{tabular}{|l|l|}
\hline Categoria de análise & Conceitos centrais \\
\hline Invenção & Teste; experimento; descoberta; novo para todos \\
\hline Difusão & Aprendizado; transferência; novo para um ator social \\
\hline Efeitos & Mudança radical em escala; amplos resultados \\
\hline
\end{tabular}

Fonte: adaptado de Jordan e Huitema (2014, p. 389)

Segundo os autores do Quadro 1, a categoria "Invenção" engloba pesquisas acerca de iniciativas nas quais os atores sociais testam e experimentam novas regras, ou novas tecnologias, de adaptação climática. Por sua vez, "Difusão" categoriza pesquisas sobre iniciativas que divulgam o aprendizado com enfoques inovadores, valorizando não apenas a criação, mas, especialmente, a adoção em escala crescente. Apesar da conotação geralmente positiva associada à ideia de inovação, a categoria "Efeitos" engloba pesquisas que analisam se a adoção de inovações gerou mudança de comportamento dos agentes econômicos. Apesar de ser recente, este referencial vem sendo integrado ao debate sobre adaptação na esfera subnacional (BAUER; STEURER, 2014; ANDERTON; SETZER, 2017).

Mudanças significativas podem ser exemplificadas com a crescente adesão, no país, ao Marco de Ação de Hyogo 2005-2015, um dos Efeitos da adaptação. Após a catástrofe da enxurrada na Região Serrana/RJ, em janeiro de 2011, e a evidência do despreparo do setor público para resposta ao desastre, o Estado reagiu com a reformulação e modernização da Defesa Civil. Pela lei n. 12.608, de 10/04/12, foi instituída nova Política Nacional de Proteção e Defesa Civil, acompanhada de recursos públicos em volume significativo para sua operacionalização por governos estaduais e prefeituras no 
PAC Prevenção. O Marco de Hyogo fomentou ainda ações em escala subnacional, como a campanha Construindo Cidades Resilientes, encontrando continuidade sob o Marco de Sendai 2015-2030 ( UNITED NATIONS OFFICE FOR DISASTER RISK REDUCTION, 2015).

No presente trabalho, buscamos sistematizar ações, e primeiros resultados, de projeto de pesquisa aplicada que busca se inserir na categoria da Difusão na inovação para adaptação climática. Inovações alteram interesses e padrões conhecidos, podendo gerar resistência, seja por desconhecimento em geral, ou por ferir interesses particulares. Inovações também trazem riscos, demandando apreciações de custo/benefício para sua adoção e difusão. Agentes diversos estão adotando inovações e assumindo riscos em ações de adaptação climática na Região Central do RS, e o presente trabalho assume que estas iniciativas merecem destaque e participação no debate sobre o desenvolvimento da região, hoje ainda marcado essencialmente por demandas de infraestrutura ou qualificação de serviços públicos.

O projeto em questão - Adaptação à Mudança Climática do Vale do Rio Pardo/RS, iniciado em 2015, pela Universidade de Santa Cruz do Sul (UNISC) - busca identificar inovações em curso na área de adaptação climática, contribuir para sua análise e maior visibilidade no debate público, ampliando o enfoque usual em estudos de caso, com foco em uma empresa inovadora (ANTINARELLI, 2013) ou sua cadeia de suprimentos (ALVES, 2014), de modo a contribuir através de diferentes formatos de divulgação e comunicação de base científica para a mobilização social em torno do tema adaptação. Tem como área de atuação a Região Central do RS, na bacia do rio Pardo, que conta com o município de Santa Cruz do Sul como polo dinâmico, sede da Universidade de Santa Cruz do Sul (UNISC), uma instituição comunitária de desenvolvimento regional. A microrregião da bacia do rio Pardo compreende 23 municípios entre as encostas da Serra Geral e as planícies da Depressão Central.

\section{Do diagnóstico à ação}

De forma precursora, entre 2013/14, pesquisa coordenada pelo Laboratório de Geologia Ambiental, da Universidade Federal de Santa Maria, mapeou os riscos de 
inundação no município de Santa Cruz do Sul (MENEZES, 2014). Os dados coletados foram utilizados em parceria com o Núcleo de Gestão Pública (NGP) da UNISC e a Defesa Civil municipal, para auxiliar na formação de Núcleos Comunitários de Defesa Civil (NUDEC) em bairros de significativa vulnerabilidade social e ambiental. Frente a relatos sobre tempestades mais intensas e mais frequentes na região, em 2015, foi iniciado o projeto de Adaptação à Mudança Climática no Vale do Rio Pardo, iniciativa em parceria com o Programa de Pós-Graduação em Desenvolvimento Regional (PPGDR) e o NGP da UNISC.

A pesquisa foi iniciada mediante diagnóstico interno, buscando identificar iniciativas correlatas em andamento na universidade e sistematizando o conhecimento produzido (Quadro 2).

Quadro 2 - síntese do conhecimento em adaptação na UNISC (ano base 2015)

\begin{tabular}{|c|c|c|}
\hline Unidade & Iniciativa & Referência \\
\hline Administração & $\begin{array}{l}\text { Mapeamento de conflitos ambientais e } \\
\text { eventos climáticos }\end{array}$ & $\begin{array}{l}\text { Bublitz; Marques, } 2016 \\
\text { Marques, } 2012\end{array}$ \\
\hline $\begin{array}{l}\text { Engenharia } \\
\text { Ambiental }\end{array}$ & $\begin{array}{l}\text { Mapeamento de áreas de risco na cidade de } \\
\text { Santa Cruz do Sul }\end{array}$ & $\begin{array}{l}\text { Pinheiro, Nummer, } \\
\text { Bressani, } 2012\end{array}$ \\
\hline $\begin{array}{l}\text { Engenharia } \\
\text { Ambiental }\end{array}$ & $\begin{array}{l}\text { Monitoramento da qualidade da água - } \\
\text { Protetor das Águas no Arroio Andréas }\end{array}$ & Oliveira et al., 2014 \\
\hline $\begin{array}{l}\text { Engenharia } \\
\text { Ambiental }\end{array}$ & $\begin{array}{l}\text { Projeto Pagamento por Serviços Ambientais } \\
\text { - uso da água no município de Vera Cruz }\end{array}$ & $\begin{array}{l}\text { Moraes, } 2012 \\
\text { Delevati; Vaz, } 2010\end{array}$ \\
\hline $\begin{array}{l}\text { Núcleo de } \\
\text { Gestão Pública }\end{array}$ & $\begin{array}{l}\text { Capacitação de voluntárias nos Núcleos } \\
\text { Comunitários de Defesa Civil }\end{array}$ & \\
\hline Diversos & $\begin{array}{l}\text { Assessoria ao Comitê de Gestão da Bacia do } \\
\text { Rio Pardo }\end{array}$ & $\begin{array}{l}\text { Delevati et al., } 2007 \\
\text { Vaz et al., } 2004\end{array}$ \\
\hline
\end{tabular}

De acordo com o Quadro 2, setores diversos da UNISC vêm atuando mediante projetos de pesquisa e de extensão em áreas como: gestão de riscos e prevenção de desastres no município de Santa Cruz do Sul; mapeamento de conflitos pelo uso de recursos naturais; gestão compartilhada dos recursos hídricos, na bacia do Rio Pardo. 
Destacamos a assessoria à prefeitura do município de Vera Cruz (cerca de 35.000 habitantes), que adotou a primeira política pública de pagamento aos agricultores produtores de água no estado, na sub-bacia do Arroio Andréas. O programa Protetor da Água foi iniciado como ação de extensão universitária, sendo integrado à legislação municipal. O serviço de água e saneamento é municipalizado e as ações de conservação de nascentes e recomposição de mata ciliar ao longo do Arroio Andréas passaram a incorporar as políticas públicas de gestão dos recursos hídricos, no âmbito da preparação do munícipio para as projeções de crescimento populacional para as próximas duas décadas. Em paralelo, foi conduzido pelo projeto um segundo diagnóstico, com base nos arquivos da Defesa Civil de Santa Cruz do Sul, listando os eventos climáticos mais significativos dos últimos anos. Estes dados foram compatibilizados com os dados do Atlas Brasileiro de Desastres Naturais - Volume Rio Grande do Sul (CENTRO UNIVERSITÁRIO DE ESTUDOS E PESQUISAS SOBRE DESASTRES, 2013).

Os diagnósticos foram utilizados para gerar dois produtos de comunicação de base cientifica. Foi produzido o Atlas de Desastres Naturais do município de Santa Cruz (BROSE, 2017), a primeira versão em formato e-book. O segundo resultado consistiu na publicação, em novembro de 2016, de um encarte de quatro páginas, nos jornais Arauto, no município de Vera Cruz, e Tribuna Popular, no município de Sinimbu. Ambos os municípios estão situados ao longo do rio Pardinho, importante afluente do rio Pardo que sofre inundações frequentes. Somando-se a tiragem dos dois jornais, chega-se a um universo de cerca de 10.000 leitores para os tópicos abordados no encarte:

- $\quad$ Desastres naturais mais frequentes na região;

- Complexos Convectivos de Mesoescala;

- $\quad$ Marco de Hyogo e o Programa Cidades Resilientes.

Os textos foram entremeados com imagens recentes de inundações e deslizamentos de terra em Santa Cruz do Sul.

Em significativa parcela das encostas da Serra Geral do RS, nos vales e escarpas ocupados pela agricultura familiar, o sinal de telefone celular ou de internet é intermitente, ou mesmo inexistente. No meio rural o rádio continua sendo veículo central 
de comunicação, o que reforça a importância da categoria profissional dos radialistas como formadores da opinião pública, e possíveis agentes de apoio em fortalecer uma cultura de prevenção de risco. Um dia antes da publicação, foi realizado seminário para jornalistas e radialistas de municípios da região. Na ocasião, foram apresentados os gestores da Defesa Civil e do Corpo de Bombeiros, bem como pesquisadores da universidade, como fonte de informação.

O encarte vem sendo, além disso, utilizado como veículo de informação e divulgação, nas ações junto aos NUDECs, bem como passou a ser distribuído em eventos de abrangência regional, como reuniões do Conselho Regional de Desenvolvimento ou do Comitê Gestor da Bacia Hidrográfica do Pardo.

Como terceiro diagnóstico, foi realizado um mapeamento das organizações inovadoras que desenvolvem ações de adaptação à mudança do clima. Foram identificadas mais de 70 organizações, desde empresas familiares, empresas de grande porte e organizações públicas, que iniciaram o ciclo de diagnóstico das emissões de gases de efeito estufa e plantaram árvores para neutralização das emissões. O principal agente inovador na região foi a Cooperativa Regional de Eletrificação Teutônia (CERTEL) que, em 2007, criou o programa Energia Verde, na bacia do rio Taquari. Este programa, que categorizamos como inovação pela Difusão, visa não apenas neutralizar as emissões da cooperativa como incentivar organizações associadas a identificar e neutralizar suas emissões. O Quadro 3 apresenta amostra de organizações que iniciaram o ciclo de neutralização.

Quadro 3 - amostra de organizações com inovações em adaptação (ano 2015)

\begin{tabular}{|l|l|}
\hline Organização & Município \\
\hline Tramontina madeiras & Encruzilhada do Sul \\
\hline Ervateria Ximango & Ilópolis \\
\hline Engepro construções & Santa Cruz do Sul \\
\hline LKC transportes & Santa Cruz do Sul \\
\hline
\end{tabular}




\begin{tabular}{|l|l|}
\hline Mercur artefatos de borracha & Santa Cruz do Sul \\
\hline Sebrae - Regional dos Vales & Santa Cruz do Sul \\
\hline Sulprint embalagens & Santa Cruz do Sul \\
\hline Altmann Contabilidade & Teutônia \\
\hline Câmara Municipal de Teutônia & Teutônia \\
\hline CERTEL cooperativa de eletrificação & Teutônia \\
\hline Cooperativa de crédito Sicredi & Teutônia \\
\hline Cooperativa Languiru - unidade de Laticínios & Teutônia \\
\hline
\end{tabular}

Este diagnóstico regional parece destoar do cenário pouco positivo identificado por Leão (2011), em pesquisa junto a grandes empresas e multinacionais sediadas na capital do estado do Rio Grande do Sul. De acordo com o autor, existe pouca disposição dessas empresas para investimento ou desenvolvimento organizacional na adaptação à mudança do clima. Ao contrário, os investimentos realizados com recursos próprios para minimizar a emissão de gases de efeito estufa, a pegada de carbono, de empresas nos Vales do Rio Pardo e Vale do Rio Taquari indica um elevado grau de predisposição para a inovação por parte dos gestores. E minimiza as preocupações, frequentemente expressas nas negociações internacionais, de que a adaptação à mudança do clima implicaria em custos abusivos que penalizariam a economia ou obrigariam a demissões em larga escala.

Este cenário positivo à inovação por agentes privados possibilitou à UNISC organizar o Fórum Empresarial pelo Clima, em parceria com o Conselho Regional de Desenvolvimento, o Sebrae Regional e a Cooperativa de Crédito Sicredi. Neste evento, em maio de 2017, empresas de portes diversos e representante do Banco Regional de Desenvolvimento do Extremo Sul (BRDE) apresentaram suas iniciativas voluntárias nas áreas de neutralização das emissões de carbono, eficiência energética e adoção de fontes renováveis de energia. Os cases podem ser categorizados tanto como inovação em adaptação pela Invenção, como pela Difusão. As palestras gravadas foram disponibilizadas na internet e sínteses delas foram integradas em encarte de quatro páginas, publicado pelo jornal Arauto, atingindo cerca de 7.000 leitores. 
Concluindo a sequência de diagnósticos e visando a sensibilizar a comunidade acadêmica, em setembro de 2017 foi organizada uma série de palestras. Ao longo de uma tarde foram apresentadas - para mais de 100 participantes, entre alunos, professores e lideranças comunitárias - palestras ao vivo, bem como apresentações gravadas no formato TED, sob o tema: Que mudanças queremos? Palestras e imagens do evento foram divulgadas nas mídias sociais e publicadas online. Neste mesmo mês, a UNISC recebeu certificado de redução de 237 toneladas de gases de efeito estufa, resultado do primeiro ano de migração para fontes renováveis no mercado livre de energia.

Assim, contrariando o default do governo estadual na implementação da Política Gaúcha de Mudança Climática, ou da potencial liderança por relevantes organizações setoriais da economia estadual, como as Federações de Indústria ou Agricultura, através das ações do projeto aqui descrito, no Vale do Rio Pardo, foi possível identificar significativa predisposição para a inovação por agentes econômicos locais e atores não governamentais. As iniciativas voluntárias podem ser mapeadas e analisadas mediante tipologia proposta, em 2014, por pesquisadores britânicos, que diferencia entre Invenção, Difusão e ampliação dos Efeitos de inovações na esfera subnacional. O mapeamento e a divulgação com base científica deste cenário pode contribuir para atender à demanda popular, identificada em estudo do Ministério da Ciência e Tecnologia, por ampliação do acesso à informação de base científica sobre mudança climática e seus impactos.

\section{Discussão e conclusões}

A restrita adoção das recomendações do IPCC por políticas nacionais de adaptação à mudança climática tem permitido a continuidade do aumento das emissões globais de gases de efeito estufa, ao mesmo tempo em que confere maior relevância à iniciativa dos atores subnacionais. Dadas suas dimensões continentais e o relativo grau de descentralização, o Brasil apresenta-se como laboratório para introdução e teste de inovações subnacionais na adaptação climática. O estado do Rio Grande do Sul recebeu destaque na bibliografia especializada por motivo de adoção de uma legislação abrangente, entre 2007 e 2010, porém, esta não se encontra em execução. Assim, as 
prioridades e políticas estaduais correntes, do Rio Grande do Sul, não respondem ao questionamento feito às ciências sociais pelo sociólogo Ulrich Beck, quanto às fontes de pressão e legitimação popular para novas políticas de adaptação climática. Antes da formulação de políticas públicas de adaptação, vem a inovação em processos e tecnologias de adaptação, processo que não depende do planejamento estatal, mas da inovação por agentes privados e organizações não governamentais.

Como resultado dos dois primeiros anos de execução de projeto de pesquisa iniciado em 2015, pela universidade comunitária UNISC, na bacia do rio Pardo, Região Central do RS, foram identificados quase uma centena de agentes econômicos nas bacias dos rios Pardo e rio Taquari, que promoveram voluntariamente Invenções ou Difusão em adaptação climática, em iniciativas como eficiência energética; alteração da fonte energética; inventário de emissões; neutralização das emissões de gases de efeito estufa ou pagamento por serviços ambientais. Diversos desses atores locais não apenas adotaram estas medidas em sua organização, como promoveram a adoção na cadeia produtiva ou em cooperação com outros agentes econômicos.

Constatou-se, assim, na região do vale do Rio Pardo, número significativo de gestores privados e públicos engajados no tema mudança climática e dispostos a correr riscos e a assumir os custos decorrentes da gradual adaptação à mudança climática, sem que essas iniciativas tenham sido mandatórias por legislação subnacional. Ou seja, foram oriundas da iniciativa inovadora local.

\section{Referências}

ALVES, A. Sustentabilidade além da fronteira empresarial: proatividade e articulação na cadeia de suprimentos. 2014. Dissertação (Mestrado em Administração) Universidade Federal do Rio Grande do Sul, Rio Grande do Sul, 2014.

ANDERTON, K.; SETZER, J. Subnational climate entrepreneurship: innovative climate action in California and São Paulo. 2017. Regional Environmental Change [online]. Disponivel em: <https://doi.org/10.1007/s10113-017-1160-2> Acesso em: 11/10/17. 
ANTINARELLI, A. Construindo a gestão estratégica sustentável: um estudo sobre a empresa Mercur S/A. 2013. Dissertação (Mestrado em Administração) Universidade de Santa Cruz do Sul, Rio Grande do Sul, 2013.

ANTUNES, V.; SANCHEZ, G. Análise das políticas estaduais de mudanças climáticas. In: ENCONTRO DA SOCIEDADE BRASILEIRA DE ECONOMIA ECOLÓGICA, 10, 17-21/09/2013, Vitória. Anais... Rio de Janeiro: Universidade Federal Rural do Rio de Janeiro, 2013.

BARBI, F.; FERREIRA, L. Governing climate change risks: subnational climate change policies in Brazil. Fudan Journal of the Humanities and Social Sciences.v. 9, n. 4, p. 589606, 2016.

BAUER, A.; STEURER, R. Innovation in climate adaptation policy: are regional partnerships catalysts or talking shops? Vienna: BOKU, 2014.

BECK, U. Climate for Change, or How to Create a Green Modernity? Theory, Culture \& Society, v. 27, n. 2-3, p. 254-266, 2010.

BODEN, T.; MARLAND, G.; ANDRES, R. Global, Regional and National Fossil-Fuel $\mathrm{CO}_{2}$ Emissions. Oak Ridge: Oak Ridge National Laboratory, 2017.

BRASIL. MINISTÉRIO DO MEIO AMBIENTE. Plano nacional de adaptação à mudança climática: estratégia geral. Brasília, 2015. v.1.

BROSE, M. Atlas dos desastres naturais de Santa Cruz do Sul: 1991-2016. Santa Cruz do Sul: EDUNISC, 2017.

BROSE, M. Quando a participação no desenvolvimento regional não prioriza só empregos: reflexões sobre a Califórnia/EUA. Revista Paranaense de Desenvolvimento IPARDES. v. 35, n. 125, 2013.

BUBLITZ, T.; MARQUES, A. Espaços, processos e relações de vulnerabilidade ambiental na cidade de Santa Cruz do Sul. Revista Eletrônica em Gestão, Educação e Tecnologia Ambiental - UFSM. v. 20, n. 1, p. 580-591, 2016.

CENTRO DE GESTÃO E ESTUDOS ESTRATÉGICOS. A ciência e a tecnologia no olhar dos brasileiros - Resumo executivo. Brasília: CGEE 2017.

CENTRO UNIVERSITÁRIO DE ESTUDOS E PESQUISAS SOBRE DESASTRES . Atlas brasileiro de desastres naturais: 1991-2012. Volume Rio Grande do Sul. Florianópolis: UFSC, 2013. 
CUNHA, K.; REI, F.; WALTER, A. Sub-national climate-friendly governance in the developing world: the case of São Paulo. In: DIAS, P. et al. (Eds.) Public policy, mitigation and adaptation to climate change in South America. São Paulo: IEA/IUSP, p. 49-73, 2009.

DELEVATI, D.; VAZ, V. Importância da preservação das nascentes em uma bacia hidrográfica. In: COSTA, A. (Org.) Água \& saúde. Santa Cruz do Sul: EDUNISC, 2010.

DELEVATI, D.; VAZ, V.; REIS, C.; DORNELLES, M. O plano da bacia do Rio Pardo. In: CONGRESSO BRASILEIRO DE ENGENHARIA SANITÁRIA E AMBIENTAL, 24, Belo Horizonte. Anais... Rio de Janeiro: ABES, 2007.

GALARRAGA, I., SAINZ DE MURIETA, E.; FRANÇA, J. Climate policy at the sub-national level. In: AVERCHENKOVA, A. et al. (Eds.) Trends in Climate Change Legislation. London: Edward Elgar, 2017, p. 143-174.

GALARRAGA, I., GONZALEZ-EGUINO, M.; MARKANDYA, A. The role of regional governments in climate change policy. Environmental Policy and Governance, v. 21, n. 164-182, 2011.

GOVERNMENT ACCOUNTABILITY OFFICE. International Climate Change Assessments : report to congress GAO-12-43. Washington, nov. 2011.

JORDAN, A; HUITEMA, D. Policy innovation in a changing climate: sources, patterns and effects. Global Environmental Change. v. 29, p. 387-394, 2014.

LEÃO, E. As respostas das empresas localizadas no RS frente às mudanças do clima. Dissertação (Mestrado em Administração) Universidade Federal do Rio Grande do Sul, Rio Grande do Sul, 2011.

MARQUES, A. Análise da paisagem e dinâmicas territoriais na bacia hidrográfica do Rio Pardo/RS. Tese (Doutorado em Ciências Biológicas) Universidade Federal de São Carlos, São Paulo, 2012.

MENEZES, D. Zoneamento das áreas de risco de inundação na área urbana de Santa Cruz do Sul. 2014. Dissertação (Mestrado em Geografia) Universidade Federal de Santa Maria, Rio Grande do Sul, 2014.

MORAES, J. PSA como instrumento de política pública: o projeto Protetor das Águas de Vera Cruz/RS. Sustentabilidade em debate. v. 3, n. 1, p. 43-56, 2012.

NOBRE, C.; YOUNG, A.; SALDIVA, P.; MARENGO, J.; ALVES JR., S; LOMBARDO, M. Vulnerabilidades das megacidades brasileiras às mudanças climáticas: São Paulo. Sumário Executivo. São José dos Campos: INPE, 2010. 
OLIVEIRA, P.; DELEVATI, D.; COSTA, A.; LOBO, E. Avaliação da qualidade da água de nascentes na bacia hidrográfica do Arroio Andréas. Revista Jovens Pesquisadores-UNISC. v. 4, p. 32-41, 2014.

PAGIOLA, S.; GLEHN, H.; TAFFARELLO, D. (Orgs.) Experiências de pagamentos por serviços ambientais no Brasil. São Paulo: Secretaria do Meio Ambiente/Banco Mundial, 2013.

PINHEIRO, R.; NUMMER, A.; BRESSANI, L. Análise da instabilidade de uma encosta localizada na área urbana em Santa Cruz do Sul. Revista Geociências. v. 31, n. 2, p. 159-174, 2012.

ROMEIRO, V.; PARENTE, V. Regulação das mudanças climáticas no Brasil e o papel dos governos subnacionais In: MOTTA, R. et al. (Eds.) Mudança do clima no Brasil: aspectos econômicos e sociais. Brasília: IPEA, 2011, p. 44-56.

SOMANATHAN, E.; STERNER, T.; SUGIYAMA, T. (Coords.) National and Subnational Policies and Institutions. In: Climate Change 2014: fifth assessment report of the IPCC. Cambridge: Cambridge University Press, 2014.

UNITED NATIONS OFFICE FOR DISASTER RISK REDUCTION. Marco de Sendai para la reducción del riesgo de desastres 2015-2030. Genebra: UNISDR, 2015.

VAZ, V.; MACHADO, A.; DELEVATI, D.; KUNRATH, I.; KAERCHER, M. Gestão das águas na bacia hidrográfica do Rio Pardo. In: SEMINÁRIO REGIONAL DE EDUCAÇÃO AMBIENTAL, 4., Santa Cruz do Sul. Anais... Santa Cruz do Sul: EDUNISC, 2004. 